\title{
Surgical management and outcomes of type A dissection-the Mayo Clinic experience
}

\author{
Alduz Cabasa, Alberto Pochettino \\ Division of Cardiovascular Surgery, Mayo Clinic, Rochester, Minnesota, USA \\ Correspondence to: Alberto Pochettino. Division of Cardiovascular Surgery, Mayo Clinic, Rochester, 200 First St SW, Rochester, MN 55905, USA. \\ Email: pochettino.alberto@mayo.edu.
}

Background: Type A aortic dissection (TAAD) is a complex cardiovascular disease that is associated with high perioperative morbidity and mortality. The most effective approach is still being debated-such as the best cannulation technique, and conservative versus extensive initial surgery. We reviewed our experience over the last 20 years and examined for variables that correlated with observed outcomes.

Methods: All patients who underwent TAAD repair were reviewed. Chi-Square tests, Fisher Exact tests and Wilcoxon tests were performed where appropriate. Survival and freedom from reoperations were analyzed with the Kaplan-Meier actuarial method.

Results: Acute TAAD was associated with a higher incidence of permanent stroke $(\mathrm{P}=0.010)$, renal failure $(\mathrm{P}=0.025)$, prolonged mechanical ventilator support $(\mathrm{P}=0.004)$, higher operative mortality $(\mathrm{P}=0.039)$ and higher 30-day mortality $(\mathrm{P}=0.003)$ compared to chronic TAAD. There was a trend towards higher risk for transient neurologic events among patients who were reoperated on $(\mathrm{P}=0.057)$. Extensive proximal repair led to longer perfusion and cross clamp times $(\mathrm{P}<0.001)$ and the need for temporary mechanical support postoperatively $(\mathrm{P}=0.011)$. More patients that had extensive distal repair underwent circulatory arrest $(\mathrm{P}=0.009)$ with no significant differences in the incidence of peri-operative complications, early, middle and long-term survival compared to the conservative management group. Overall survival in our series was $66.73 \%$ and $46.30 \%$ at 5 and 10 years respectively (median survival time: 9.38 years). There was a significant improvement in operative mortality $(\mathrm{P}=0.002)$ and 30-day mortality $(\mathrm{P}=0.033)$ in the second decade of our study.

Conclusion: TAAD is a complex disease with several options for its surgical management. Each technique has its own advantages and complications and surgical management should be individualized depending on the clinical presentation. We propose our present approach to maximize benefits in both the short and long term.

Keywords: Ascending aorta dissection; type A dissection; DeBakey I dissection; surgical repair; arterial cannulation; root management

Submitted May 16, 2016. Accepted for publication Jun 02, 2016.

doi: 10.21037/acs.2016.06.01

View this article at: http://dx.doi.org/10.21037/acs.2016.06.01

\section{Introduction}

Ascending aortic dissection remains one of the most catastrophic emergencies amongst cardiovascular diseases (1-5). Delay in diagnosis and surgical treatment is associated with increased mortality $(2,6)$. Collective efforts from different groups in the last 20 years have aimed to improve outcomes, but there are still many aspects in its management that have yet to be agreed upon $(7,8)$. These include the most efficient cannulation technique, appropriate aortic root management, and extent of distal repair. Despite diminishing reliance on the femoral artery, there has been no consensus on the best arterial cannulation technique with the lowest complication rate that could shorten operative times (9). Proponents of a more conservative root management and extent of distal repair 
claimed that early survival is improved by reducing the complexity of the surgery, a gain that is offset by the higher risk of reoperation when diseased aortic tissue is left in situ $(1,7,8,10)$.

The objective of this study is to review our experience with management of TAAD over the last two decades. We aim to answer these questions by looking at the early and late outcomes for each technique.

\section{Methods}

We retrospectively reviewed the medical records of all patients diagnosed with TAAD, admitted and surgically managed in our institution from 1993 to 2014. Variables were collected from electronic records and from the Society of Thoracic Surgeons (STS) database at Mayo Clinic. This study was approved by the Institutional Review Board at Mayo Clinic, Rochester MN.

Continuous variables were expressed as mean $\pm \mathrm{SD}$ and categorical variables were expressed as count (percentage). Chi-Square test, Fisher's Exact test, and Wilcoxon test were utilized to find baseline and postoperative differences between groups where appropriate. Survival curves were estimated with the Kaplan-Meier product limit method and univariate group comparisons were based on the logrank test. To estimate the effect of redo-thoracic aorta repair, first time repair was considered as a time dependent variable in the Cox regression model to allow patients with reoperations at our facility to contribute to both first time and re-repair groups. A P value $<0.05$ was considered statistically significant. Data analysis was performed using SAS statistical analysis tool (Version 9.4 SAS Institute Inc., Cary, NC, USA).

\section{Surgical technique}

Depending on the clinical presentation at the ED, patients either underwent stat preoperative imaging or were brought directly to the operating room. Patients were repaired through a median sternotomy, with full heparinization therapy under cardiopulmonary bypass (CPB). Distal repair was performed either with an open technique under hypothermic circulatory arrest or with cross clamping of the distal ascending aorta, and with or without cerebral perfusion, based on the extent of the planned repair and surgeon's preference. Arterial cannulation was accessed either through the femoral, axillary, innominate artery or ascending aorta. Venous drainage was secured either through the femoral vein, or the right atrium with a twostage venous cannula or via a bicaval approach. A left ventricular vent catheter was usually placed through the right superior pulmonary vein.

Once CPB was initiated, patients were systemically cooled. If an open distal repair was chosen, the patients were core cooled to perform the distal repair under hypothermic circulatory arrest. Myocardial protection strategy utilized antegrade cardioplegia or a combination with retrograde cardioplegia. Depending on the surgeon preference and availability in the emergency setting, intra-operative electroencephalograms (EEG) were used to monitor cerebral function and determine the timing for circulatory arrest. If not available, patients were core cooled for at least 45 minutes and/or until the desired core temperature was reached. The root was either managed conservatively with options such as valve resuspension, suture repair of the dissected aortic root wall, or with a combination of aortic valve replacement and ascending aortic replacement (Wheat procedure), or a more extensive strategies for repair, such as a root replacement with a valved conduit, or a valve sparing root replacement (using David's, Yacoub's or a partial root remodeling technique). Open distal aortic arch anastomosis was performed under circulatory arrest. Total arch replacement was performed if the arch measured more than $5 \mathrm{~cm}$ in diameter, in the case of a complex arch tear, or in arch rupture. For patients undergoing total arch replacement, the branch vessels were preserved as a single aortic cuff off the transverse arch or individually reattached during the procedure. Once the distal reconstruction was completed, the graft was deaired and antegrade perfusion was resumed by cannulating the new Dacron graft, or through the axillary or innominate artery if they had been used for arterial access. Systemic rewarming was then initiated, and the proximal reconstruction was completed.

\section{Results}

\section{Demographic data, comparison of two eras}

There were 375 patients who met our inclusion criteria in the last 20 years (Figure 1A). Our median clinical follow up time was $7.42(6.03-8.75)$ years. We averaged 18.75 patients/year with a slow upward trend: 164 patients in the first half and 211 in the latter. There were more males $(\mathrm{P}=0.041)$ and a lower incidence of peripheral arterial disease $(\mathrm{P}<0.001)$ in the second half, but preoperative clinical characteristics were otherwise similar (Table 1). 
A

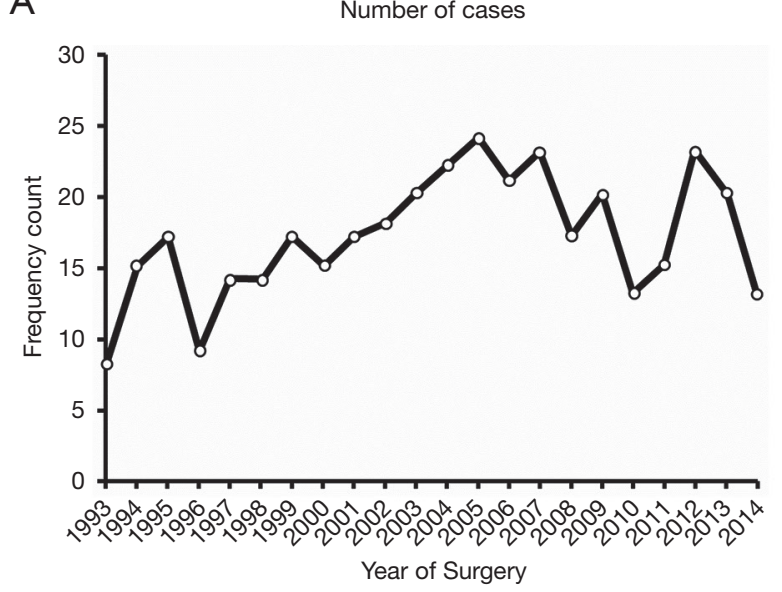

C

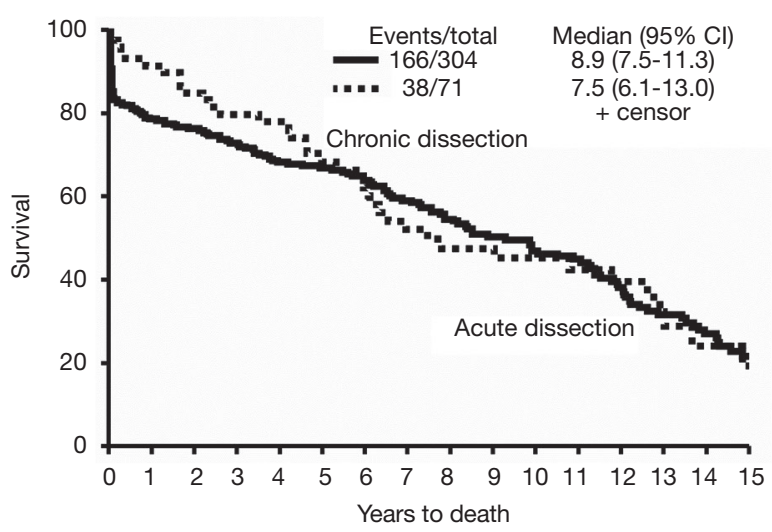

$\mathrm{E}$

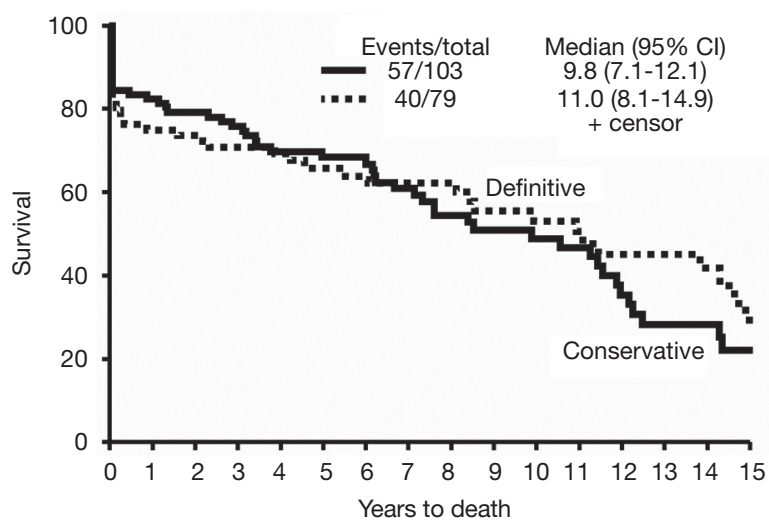

B Survival for all cases

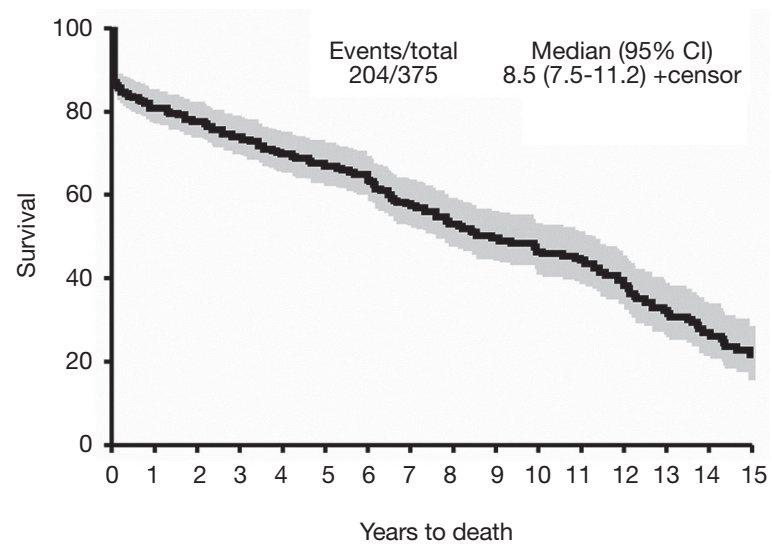

D

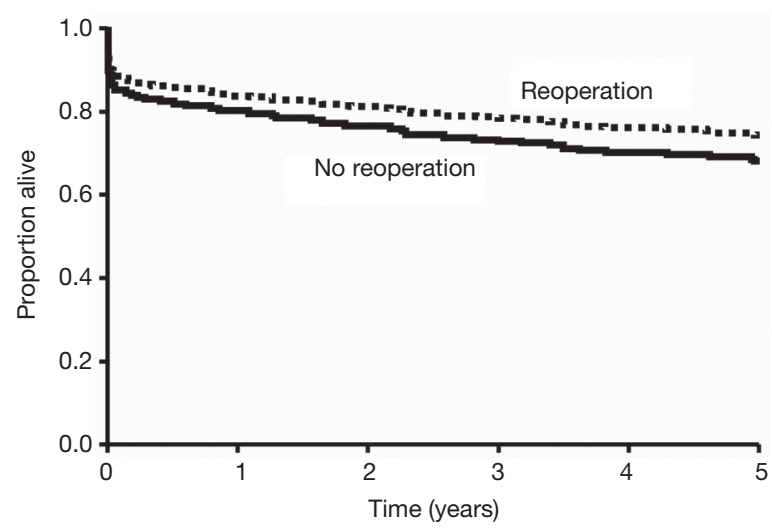

$\mathrm{F}$

Survival by extent of distal repair

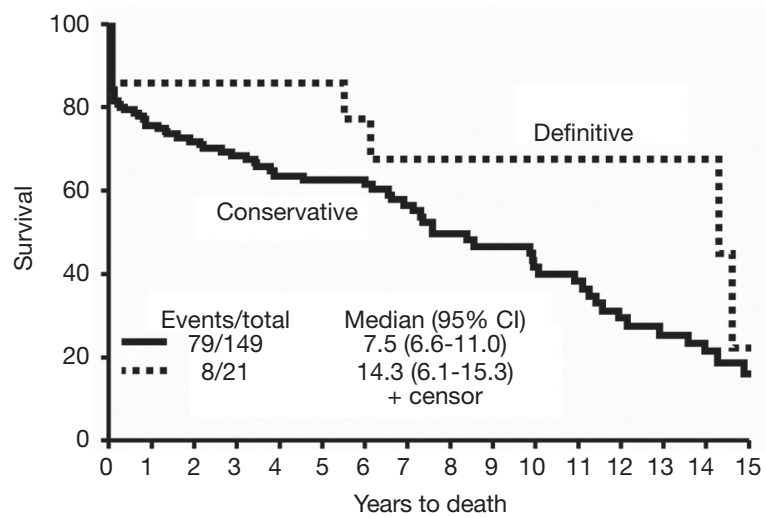

Figure 1 Survival curves. (A) Line graph of the annual census for TAAD patients in the last 20 years; (B) overall survival of TAAD patients in our series; (C) Kaplan-Meier survival curve comparing acute and chronic TAAD; (D) survival curve comparing first time surgery patients versus reoperation patients, with reoperation considered as time dependent variable; (E) Kaplan-Meier survival curve comparing conservative versus definitive root management during the initial surgery for TAAD; (F) Kaplan-Meier survival curve comparing conservative versus extensive extent of distal repair during the initial surgery for TAAD. CI, confidence interval. 
Table 1 Demographic data, analysis by decade, and 30-day mortality risk factors

\begin{tabular}{|c|c|c|c|c|c|}
\hline Variable* & $1^{\text {st }}$ decade $(\mathrm{N}=164)$ & $2^{\text {nd }}$ decade $(\mathrm{N}=211)$ & $P$ value & $\begin{array}{l}\text { Risk factor for early } \\
(30 \text { d) mortality }(\mathrm{N}=52)\end{array}$ & $P$ value \\
\hline \multicolumn{6}{|l|}{ Pre-operative variables } \\
\hline Age (years) & $65.0 \pm 13.8$ & $65.1 \pm 14.5$ & 0.932 & $70.7 \pm 15.2$ & $<0.001$ \\
\hline$\leq 45$ & $18(11.0 \%)$ & $21(20.0 \%)$ & 0.308 & $5(9.6 \%)$ & 0.002 \\
\hline $46-60$ & $31(18.9 \%)$ & $54(25.6 \%)$ & & $3(5.8 \%)$ & \\
\hline$>60$ & $115(70.1 \%)$ & $136(64.5 \%)$ & & $44(84.6 \%)$ & \\
\hline Male & $101(61.6 \%)$ & $151(71.6 \%)$ & & $35(67.3 \%)$ & \\
\hline Female & $63(38.4 \%)$ & $60(28.4 \%)$ & & $17(32.7 \%)$ & \\
\hline $\mathrm{BMI}$ & $27.2 \pm 5.1$ & $28.5 \pm 6.6$ & 0.173 & $27.1 \pm 4.7$ & 0.485 \\
\hline Smoker & $96(58.9 \%)$ & $104(49.3 \%)$ & 0.065 & $25(49 \%)$ & 0.546 \\
\hline Diabetes & $12(7.4 \%)$ & $25(11.8 \%)$ & 0.150 & $7(13.7 \%)$ & 0.319 \\
\hline Peripheral arterial disease & $45(28.1 \%)$ & 27 (12.9\%) & $<0.001$ & $7(14.9 \%)$ & 0.438 \\
\hline Cerebrovascular disease & $30(18.9 \%)$ & $37(17.7 \%)$ & 0.773 & $11(23.9 \%)$ & 0.301 \\
\hline First time repair & $156(95.1 \%)$ & $195(92.4 \%)$ & 0.288 & $50(96.2 \%)$ & 0.553 \\
\hline \multicolumn{6}{|l|}{ Re-repair } \\
\hline Previous CABG & $19(11.6 \%)$ & $30(14.2 \%)$ & 0.453 & $7(13.5 \%)$ & 1.000 \\
\hline Previous valve surgery & $21(12.8 \%)$ & $30(14.2 \%)$ & 0.692 & $7(13.5 \%)$ & 1.000 \\
\hline \multicolumn{6}{|l|}{ Clinical presentation } \\
\hline Tamponade & $49(29.9 \%)$ & $42(19.9 \%)$ & 0.025 & $19(36.5 \%)$ & 0.036 \\
\hline $\mathrm{CHF}$ & $15(9.4 \%)$ & $18(8.6 \%)$ & 0.774 & $7(14.6 \%)$ & 0.123 \\
\hline Reoperation for bleeding & $13(7.9 \%)$ & $16(7.7 \%)$ & 0.927 & $4(8.0 \%)$ & 1.000 \\
\hline Sternal infection (superficial) & $2(1.2 \%)$ & $5(2.4 \%)$ & 0.473 & $1(2.01 \%)$ & 1.000 \\
\hline Sternal infection (deep) & $1(0.6 \%)$ & $1(0.5 \%)$ & 1.000 & 0 & 1.000 \\
\hline Stroke & $15(9.1 \%)$ & $18(8.6)$ & 0.857 & $13(26.0 \%)$ & $<0.001$ \\
\hline TIA & $5(3.0 \%)$ & $3(1.4 \%)$ & 0.307 & 0 & 0.605 \\
\hline ARDS & $2(1.2 \%)$ & $1(0.5 \%)$ & 0.590 & $1(2.0 \%)$ & 0.360 \\
\hline Pneumonia & $11(6.7 \%)$ & $19(9.1 \%)$ & 0.448 & $2(4.0 \%)$ & 0.402 \\
\hline Prolonged ventilator support & $63(38.4 \%)$ & $93(44.5 \%)$ & 0.237 & $20(40.0 \%)$ & 0.761 \\
\hline Renal failure & $23(14.0 \%)$ & $23(11.0 \%)$ & 0.379 & $13(26.0 \%)$ & 0.004 \\
\hline Complete heart block & $4(2.4 \%)$ & $4(1.9 \%)$ & 0.735 & 0 & 0.605 \\
\hline GI complications & $7(4.3 \%)$ & $11(5.3 \%)$ & 0.656 & $4(8.0 \%)$ & 0.283 \\
\hline Operative mortality & $19(11.6 \%)$ & $7(3.3 \%)$ & 0.002 & $26(50.0 \%)$ & $<0.001$ \\
\hline 30 day mortality & $30(18.3 \%)$ & $22(10.6 \%)$ & 0.033 & & \\
\hline
\end{tabular}


The majority of patients were at least 60 years of age in both time periods. There was a significant improvement in operative mortality (defined as mortality within one day of surgery) $(\mathrm{P}=0.002)$, and 30-day mortality $(\mathrm{P}=0.033)$ in the second half of our study. Longer cross clamp time, perfusion time, and more cases done under circulatory arrest characterized the last ten years. The presence of pre-operative cardiac tamponade was associated with higher early mortality $(\mathrm{P}=0.036)$, and was significantly lower in the second decade $(\mathrm{P}=0.025)$, suggestive of earlier diagnosis. Age $(\mathrm{P}<0.001)$, development of postoperative stroke $(\mathrm{P}<0.001)$ and renal failure $(\mathrm{P}=0.004)$ were associated with increased 30 -day mortality. The overall survival in our series was $66.73 \%$ and $46.30 \%$ at 5 and 10 years respectively, with a median survival time of 9.38 years (Figure 1B).

\section{Acute versus chronic TAAD and primary repair versus reoperations}

The effects of the dissection chronicity on post-operative outcomes were examined. Acute dissection was defined as $\leq 14$ days from onset of symptoms/dissection and chronic if $>14$ days or with intraoperative findings suggestive of long standing dissection (i.e., thrombus formation in the false lumen) (Table 2). More patients were in heart failure (NYHA Class $\geq$ III) in the acute dissection group $(\mathrm{P} \leq 0.001)$, but there was no difference in the severity of preoperative aortic regurgitation (AR). More patients in the chronic dissection group had at least one previous heart surgery $(\mathrm{P} \leq 0.001)$. More acute dissection patients were amenable to valve resuspension with preservation of the aortic root wall, although this did not reach statistical significance $(\mathrm{P}=0.055)$. Patients with chronic dissection underwent more extensive distal repair (total arch $\mathrm{P}=0.027$, proximal descending $\mathrm{P}=0.032$ ). Acute dissection was associated with worse perioperative outcomes: permanent stroke $(\mathrm{P}=0.010)$, renal failure $(\mathrm{P}=0.025)$, need for prolonged mechanical ventilator support $(\mathrm{P}=0.004)$, higher operative mortality $(\mathrm{P}=0.039)$ and 30-day mortality $(\mathrm{P}=0.003)$. However, there was no significant difference in the middle and long-term survival between the two groups (Figure 1C).

There were 47 patients who underwent reoperations: 24 had their first repair done elsewhere and underwent redo-surgery at our institution, while the other 23 had both repairs done at our facility (Table 2). Since data on the initial surgeries of the former group were not available, they were excluded in some of the statistical analyses. Patients undergoing primary repair had worse functional class $(\mathrm{P}<0.001)$ and longer perfusion time $(\mathrm{P}=0.012)$ compared to patients undergoing second (or third) repair. There was a trend towards higher risk of transient neurologic events amongst redo-repair patients $(\mathrm{P}=0.057)$. There were no other significant differences between the two groups in terms of peri-operative complications. In our series, the median time from the initial surgery to reoperation was 3.52 (range, 0.12-13.27) years. By considering reoperation as a time-dependent variable, we compared the survival curves of first time surgery versus patients undergoing reoperation (Figure 1D). There were a total of 397 surgeries and 167 deaths by the time of last clinical followup. Reoperation was shown to be slightly protective, with a lower patient mortality rate after a redo repair compared to those who only underwent one surgery, although this was not statistically significant [HR $0.76,95 \%$ CI reoperation (0.44, 1.33), $\mathrm{P}=0.32]$.

\section{Cannulation technique}

There were four common arterial cannulation sites in our series: femoral artery (51.5\%), central/aortic (27.5\%), axillary $(19.7 \%)$, and innominate $(1.3 \%)$ artery, with the innominate artery cannulation associated with longer perfusion $(\mathrm{P}=0.012)$ and cross clamp time $(\mathrm{P}=0.008)$ (Table 3). There were no significant differences between the four techniques in terms of stroke rate, renal failure, or GI complications. Thirty-day mortality and length of hospital stay were not affected by the cannulation technique used. Interestingly, the majority of patients in the first half of our study underwent femoral cannulation (74.4\%), while in the second decade, there was a shift to more variable approaches (aortic $31.3 \%$, femoral $33.7 \%$, axillary $32.7 \%$, innominate $2.4 \%$ ). Femoral artery was the most common cannulation site for acute dissection cases (55.9\%), while aortic cannulation was most commonly performed amongst patients presenting with chronic dissection (40.9\%). There was only one documented case of axillary cannulation site infection in our series.

We looked at factors that might be predictive of postoperative neurologic complications (Table 4). Acuteness of the dissection was the only significant factor noted $(\mathrm{P}=0.015)$. Age, cross clamp time, circulatory arrest time of more than 20 minutes, and arterial cannulation approach were not associated with neurological complications. Detailed data on cerebral perfusion during circulatory arrest were unfortunately not available in our series. 


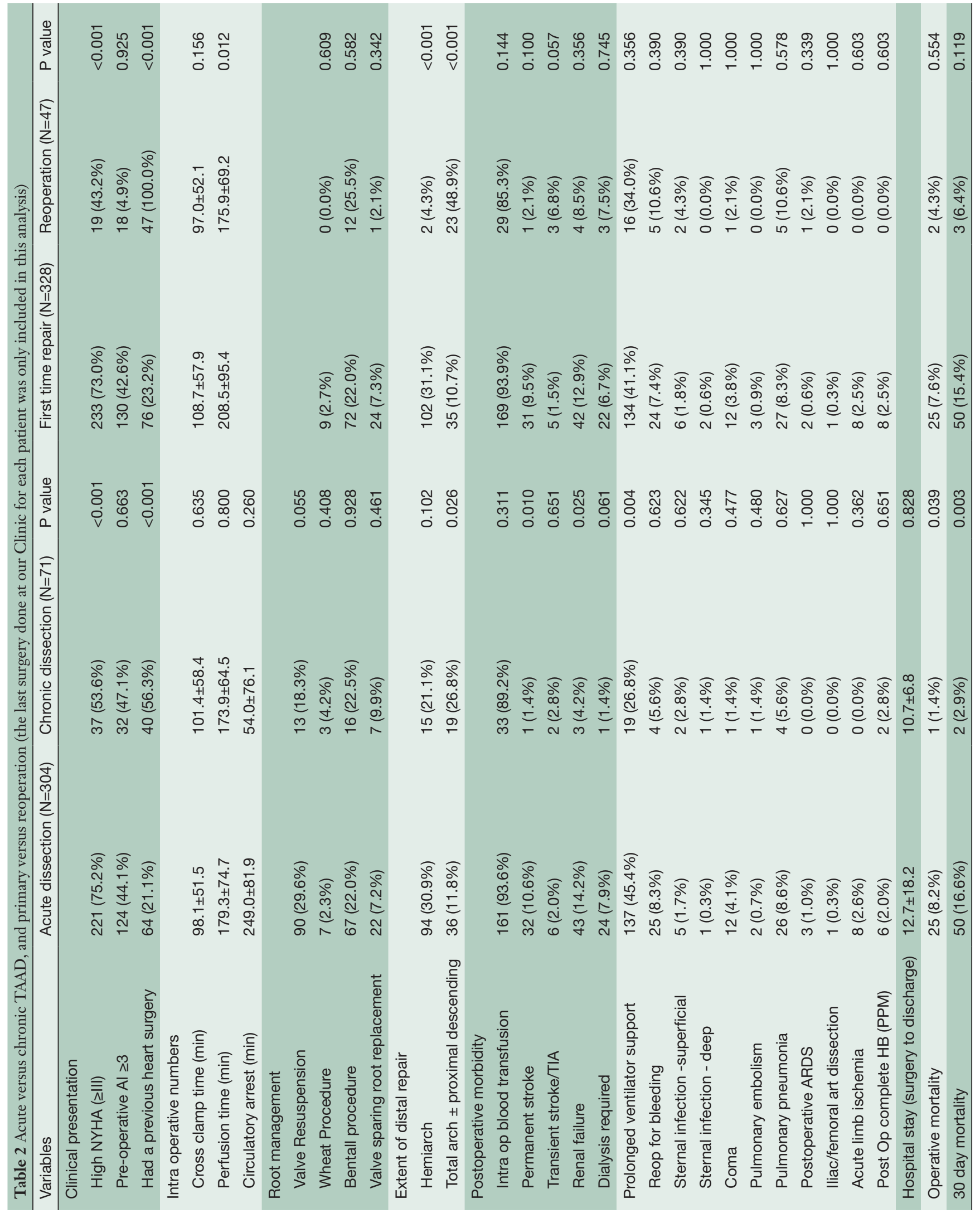




\begin{tabular}{|c|c|c|c|c|c|}
\hline Variables & Axillary $(\mathrm{N}=74)$ & Central $(\mathrm{N}=103)$ & Femoral $(\mathrm{N}=193)$ & Innominate $(\mathrm{N}=5)$ & $P$ value \\
\hline Total perfusion time (min) & $194.4 \pm 56.7$ & $176.3 \pm 89.8$ & $171.3 \pm 65.7$ & $247.4 \pm 107.7$ & 0.012 \\
\hline Cross clamp time (min) & $107.4 \pm 47.6$ & $105.1 \pm 63.7$ & $89.8 \pm 43.5$ & $176.8 \pm 102.7$ & 0.008 \\
\hline Circulatory arrest (N, \%) & $71(95.9 \%)$ & $72(69.9 \%)$ & $155(80.3 \%)$ & $5(100.0 \%)$ & $<0.001$ \\
\hline Renal failure & $6(8.2 \%)$ & $12(11.8 \%)$ & $28(14.5 \%)$ & $0(0.0 \%)$ & 0.525 \\
\hline Dialysis required & $5(6.8 \%)$ & $9(8.8 \%)$ & $11(5.7 \%)$ & $0(0.0 \%)$ & 0.701 \\
\hline Gl complications & $1(1.4 \%)$ & $5(4.9 \%)$ & $12(6.2 \%)$ & $0(0.0 \%)$ & 0.435 \\
\hline Transient stroke/TIA & $1(1.4 \%)$ & $2(2.0 \%)$ & $5(2.6 \%)$ & $0(0.0 \%)$ & 1.000 \\
\hline Permanent stroke & $5(6.8 \%)$ & $8(7.8 \%)$ & $19(9.8 \%)$ & $1(20.0 \%)$ & 0.529 \\
\hline 30 day mortality & $9(12.3 \%)$ & $11(10.8 \%)$ & $32(16.7 \%)$ & $0(0.0 \%)$ & 0.483 \\
\hline Hospital stay (surgery to discharge) & $9.6 \pm 5.8$ & $13.6 \pm 21.4$ & $12.7 \pm 16.7$ & $12.2 \pm 1.8$ & 0.301 \\
\hline
\end{tabular}

\begin{tabular}{|c|c|c|c|}
\hline Variables & No neurological complications $(\mathrm{N}=302)$ & With neurological complications $(\mathrm{N}=71)$ & Logistic ( $\mathrm{P}$ value) \\
\hline Age (mean) & 67.7 & 68.7 & 0.689 \\
\hline$\leq 45$ & $32(10.6 \%)$ & $7(9.9 \%)$ & \\
\hline $46-60$ & $69(22.8 \%)$ & $16(22.5 \%)$ & \\
\hline $\mathrm{CHF}$ in the last 2 weeks & $28(9.4 \%)$ & $4(5.7 \%)$ & 0.330 \\
\hline Peripheral arterial disease & $54(18.1 \%)$ & $18(25.7 \%)$ & 0.148 \\
\hline Cerebrovascular disease & $54(18.1 \%)$ & $13(18.6 \%)$ & 0.930 \\
\hline Acute type A dissection & $237(78.5 \%)$ & 65 (91.5\%) & 0.015 \\
\hline Circ arrest time $>20 \mathrm{~min}$ & $187(61.9 \%)$ & $43(60.6 \%)$ & 0.832 \\
\hline Arterial cannulation & & & 0.701 \\
\hline Central & $86(28.5 \%)$ & $16(22.5 \%)$ & \\
\hline Axillary & 60 (19.9\%) & $13(18.3 \%)$ & \\
\hline Femoral & $152(50.3 \%)$ & $41(57.7 \%)$ & \\
\hline Other & $4(1.3 \%)$ & $1(1.4 \%)$ & \\
\hline
\end{tabular}

\section{Extent of proximal and distal repair}

There were 182 cases of first time repair for acute TAAD with root involvement (Table 5). Surgical option was defined as either conservative (103 cases, $56.6 \%$ ) wherein the aortic root wall was preserved, or extensive (79 cases, $43.4 \%$ ) wherein the root was replaced with a prosthetic graft. For those with a conservative approach, 78 (75.7\%) had valve re-suspension, 14 (13.6\%) had some aortic root wall repair with tacking sutures, and three (2.9\%) patients underwent aortic valve replacement with supracoronary ascending aorta replacement (Wheat procedure). For the extensive root repair group, 58 (73.4\%) underwent root replacement with 
Table 5 Outcome of conservative versus extensive root and distal repairs in first time repair acute TAAD patients

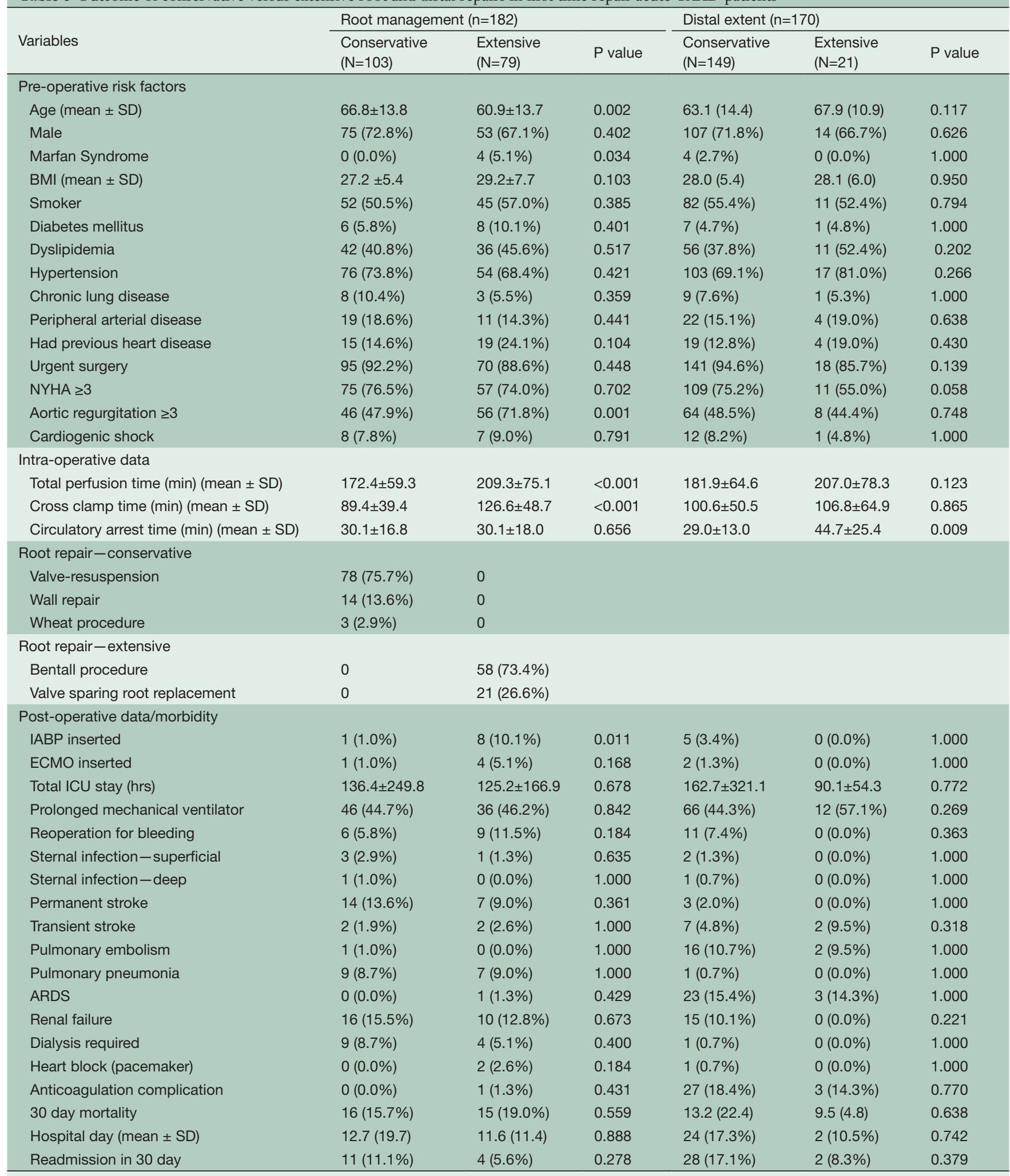

TAAD, type A aortic dissection; SD, standard deviation; IABP, intra-aortic balloon pump; ECMO, extra-corporeal membrane oxygenation; ICU, intensive care unit; ARDS, acute respiratory distress syndrome. 


\begin{tabular}{llll}
\hline \multicolumn{5}{l}{ Table 6 Etiology of early and late mortality } \\
\hline Etiology & $\begin{array}{l}\text { Early mortality } \\
(<30 \mathrm{~d})(\mathrm{N}=52)(\%)\end{array}$ & $\begin{array}{l}\text { Late mortality } \\
(>30 \mathrm{~d})(\mathrm{N}=144)(\%)\end{array}$ & P value \\
\hline Cardiac & $34(65.4)$ & $21(14.6)$ & $<0.001$ \\
\hline Neurologic & $7(13.5)$ & $6(4.2)$ & 0.052 \\
\hline Renal & $3(5.8)$ & $1(0.7)$ & 0.064 \\
\hline Vascular & $4(7.7)$ & $2(1.4)$ & 0.051 \\
\hline Infection & $1(1.9)$ & $9(6.3)$ & 0.458 \\
\hline Pulmonary & $2(3.8)$ & $8(5.6)$ & 1.000 \\
Valvular & 0 & $2(1.4)$ & 1.000 \\
\hline Malignancy & 0 & $7(4.9)$ & 0.194 \\
\hline $\begin{array}{l}\text { Unknown/ } \\
\text { others }\end{array}$ & $1(1.9)$ & $88(61.1)$ & $<0.001$ \\
\hline
\end{tabular}

a valved conduit, while 21 (26.6\%) had valve-sparing root replacement (VSRR). This last subset was subdivided into $6(7.4 \%)$ who underwent valve re-implantation (David's) procedures, five $(6.2 \%)$ who had root remodeling (Yacoub's), and $10(12.3 \%)$ who had partial root reconstructions (NCC patch repair). None of the 21 VSRR patients required AVR as of their last clinical follow up.

The conservative approach was performed on relatively older patients $(\mathrm{P}=0.002)$. Extensive repair was preferred for patients with Marfan syndrome $(\mathrm{P}=0.034)$ and for those with higher pre-operative AR $(\mathrm{P}=0.001)$. More extensive repairs were associated with longer perfusion and cross clamp times $(\mathrm{P}<0.001)$. Extensive repair increased the likelihood of requiring temporary mechanical support postoperatively $(\mathrm{P}=0.011)$. There were no other significant differences in terms of pre-operative characteristics and perioperative complications, as well as the early, middle and long-term survival between the groups (Figure 1E).

Four of our 103 (3.9\%) first time repair patients with conservative root management (four valve resuspensions) in the initial surgery eventually underwent root replacement due to progressive AR, while two of 79 (2.5\%) patients who had extensive root surgery required reoperation, one due to prosthesis endocarditis and the other for pseudoaneurysm formation. There was no significant difference in reoperation rate between the two groups $(\mathrm{P}=0.700)$

For patients with dissection distal to the aortic arch (acute DeBakey I), repair can be conservative or extensive. We defined conservative as limited proximal arch repair and with a variable amount of dissected arch tissue left in situ either with distal ascending anastomosis or with a "hemiarch" technique. Extensive repairs involved replacement of all dissected aortic arch by performing some version of "total arch replacement". Based on these definitions, there were $149(87.6 \%)$ conservative first time replacements, and $21(12.3 \%)$ total arch replacements. The two groups had similar pre-operative characteristics. More patients with the extensive distal approach underwent circulatory arrest $(\mathrm{P}=0.009)$ with no significant differences in the incidence of peri-operative complications, or early, middle and long-term survival between the two groups (Figure 1F).

\section{Etiology of mortality}

Early mortalities were due to cardiac etiology $(\mathrm{P}<0.001)$ (Table 6). Neurologic and vascular complications were also contributory but they were less significant. Late mortality was usually secondary to valve pathology and malignancy. Unfortunately, there were a significant number of late mortalities in our series with undocumented etiology. The overall survival in our series was $66.73 \%$ and $46.30 \%$ at 5 and 10 years respectively (median: 9.38 years).

\section{Discussion}

Ascending aortic dissection is a less common but complex cardiovascular pathology affecting approximately 3.5 per 100,000 persons per year (11). Contemporary reports showed $10 \%$ to $25 \%$ perioperative mortality in spite of advances in techniques and perioperative management $(3-5,12)$. These numbers increase dramatically when there is hemodynamic compromise at presentation $(1,2,5-6,11,13,14)$. Hence, early recognition and surgical correction is recommended to prevent aortic rupture, repair the aortic regurgitation, and restore flow to end-organs $(2,14)$.

We have observed an increased number of TAAD in the last 20 years. Pre-operative clinical characteristics of patients have remained constant, except for a significant decrease in the incidence of cardiac tamponade. This can be an indication of improvement in early detection and recognition of the disease. Although a concrete correlation has not been analyzed, it contributes to a lower operative and 30-day mortality in the second half of our study. Advanced age, long CPB, post-operative stroke, and renal failure increased the 30-day mortality in our series.

\section{Acute versus chronic TAAD}

Chronic TAAD is a distinct group with different presentation and surgical outcomes, and should be analyzed 
separately from acute TAAD cases. Reviews have been mostly limited to case reports, and there has been no large series to guide clinical decision making (15). We operated on $71(18.9 \%)$ patients who presented with chronic dissection. This relatively higher prevalence is reflective of more elective and complex patients being referred to tertiary referral centers like ours. Compared to their acute counterparts, they tended to have a better pre-operative NYHA class and the majority had previous cardiac surgery. There was a trend towards a worse degree of pre-operative $\mathrm{AR}$ and more limited distal extent of dissection. Progressive AR secondary to gradual dilatation of the proximal aorta is the primary lesion leading to symptoms in chronic TAAD $(16,17)$. Due to greater involvement of the root, they were less amenable to simpler repairs and usually required more extensive root replacements. In spite of this, they showed lower incidence of postoperative stroke, renal failure, prolonged ventilator support, and overall better operative and 30-day mortality, probably due to a lack of malperfusion syndromes or cardiogenic shock.

\section{Primary repair and reoperation}

With the improving outcomes of TAAD repair, more patients are undergoing reoperations after a conservative initial surgery. Similar to early reports, aneurysmal progression of the false lumen is the most common reason for re-operation in our series $(18,19)$. Although reoperations can be challenging and significantly increase potential morbidity and mortality, the trend for most surgeons continues to favor a more limited initial repair with the plan to refer late sequelae to a specialized aortic center at later time (18-20). Leaving residual dissected aorta within the ascending and arch segments in situ will lead to late aneurysmal degeneration $(19,20)$.

Disease progression after conservative repair does not occur immediately $(18,21,22)$. Aortic growth is usually gradual and takes several years before re-operation: 3.5 years in our series, 4.7 years in Concistre's, 5.9 years in Kobuch's, and 7.7 years in Preventza's report $(18,21,22)$. There were more extensive distal repairs in our re-operations group. In spite of this, cross-clamp time and perfusion time were significantly lower, probably due to the elective nature of such interventions. There was a trend towards higher risk for transient neurologic events, otherwise perioperative outcomes were the same between the primary operated and the re-operative group. Our Cox regression analysis has shown that redo-surgery was slightly protective with fewer patients dying after redo-surgery. All these findings support that complex reoperations for repaired acute TAAD can be performed safely and thus re-operative risk alone should not dictate the operative strategy during the initial TAAD repair $(18,21)$. However, the patients who underwent elective reoperation after previous "incomplete" acute dissection repairs are a very select group, and it is likely that many more patients with dissecting aneurysms are not offered further surgery either because they are not deemed operable or because the patients accept the inevitability of their premature death due to aortic related complications. While detailed causes of death are not available for most, the poor 5 and 10-year survival among patients who have undergone repair of TAAD is likely related to the residual dissected aorta. It is telling that survival for type $\mathrm{B}$ dissection survivors managed medically parallels the number reported after repairs of type A dissection.

\section{Cannulation technique}

With each strategy offering different advantages and inherent complications, the optimal cannulation for CPB is still debated. Patient's hemodynamic stability, extent of dissection, level of the planned distal repair and surgeon's preference are some of the selection variables that determine the choice $(9,22,23)$.

Femoral artery cannulation was the first approach used in patients with aortic dissection and arch aneurysms. It is relatively rapid to access, but it is associated with retrograde debris embolization, further dissection of intimal flap, expansion of the false lumen, and potential organ malperfusion $(9,23)$. Axillary artery cannulation has the advantage of delivering cerebral perfusion through the initial cannulation site. There is less risk for visceral malperfusion, but it can be technically demanding in large patients, requires a separate incision, has been associated with brachial plexus injury and arm ischemia, and can cause insufficient systemic CPB flow if the vessel is small, has a serpentine course, atherosclerotic, or is itself dissected $(9,13,23,24)$. Innominate artery cannulation, first described by Banbury and Cosgrove in 2000, is less commonly used (23). Advantages includes avoidance of brachial plexus injury and arm ischemia, lower risk of embolic stroke, no requirement for an additional incision, and ability to be used to resume antegrade flow after circulatory arrest (23). However, innominate artery dissection is very commonwith a reported incidence as high as $70 \%$ among acute TAAD, making its use treacherous. Direct ascending aorta 
cannulation has been associated with a lesser incidence of perioperative complications, shorter operation time, CPB time, mean intubation time and mean ICU stay (9). However, it is associated with theoretical risk of aortic rupture during insertion of the aortic cannula $(9,23)$. In our series, there was no significant difference in postoperative complications among the different arterial cannulation sites. Innominate cannulation, however, was associated with longer perfusion and clamp time. There was only one incidence of axillary cannulation site infection, but this is relatively common in groin incisions after femoral artery cannulation, especially in high BMI patients, or in emergent peripheral cannulation to treat cardiovascular collapse.

\section{Root management and extent of distal repair}

Conservative root repair has been recommended to reduce the complexity of the surgery, with the goal of improving the initial operative outcome rather than long-term sequelae $(1,3,7-8,10,25,26)$. Aortic valve re-suspension has been utilized to repair the AR with good results, as long as the root is not excessively dilated or the intimal tear does not extend below the sino-tubular junction $(1,7,26)$. In contrast, others have proposed a more extensive aortic root replacement during the initial surgery, since leaving diseased tissue in situ poses a risk of aortic dilatation and pseudoaneurysm formation in as many as $9 \%$ to $27 \%$ of TAAD $(1,3,21)$.

Our suggestion is that valve re-suspension should continue to be the primary reparative technique in TAAD, often combined with obliteration or replacement of the non-coronary sinus, which is the most commonly dissected (Figure 2A,B). Root replacement should be reserved to excessively dilated roots or when the intima is deeply disrupted by the dissection process. VSRR in the reimplantation technique can be utilized by experienced aortic surgeons in young, hemodynamically stable patients and in patients without malperfusion syndromes.

The extent of distal repair in acute TAAD is even more controversial. The majority of surgeons adopt a conservative approach with the stated primary goal of achieving a good early survival (27). Except for very limited DeBakey II dissections, replacement of only the ascending aorta around a distal cross-clamp should be avoided as it carries a high risk of distal anastomotic disruption and malperfusion of the aortic arch vessels, leading to strokes (27-29). While most surgeons remain fearful of hypothermic cardiac arrest, a standard hemiarch replacement is the safest and most effective method to secure a solid distal anastomosis that will improve true lumen flow downstream, and minimize the risk of aortic arch vessels malperfusion (Figure 2C,D) $(7,27)$. Such an approach, however, leaves the patient with a residual patent "type B" dissection with similar long term outcomes to medically managed standard type B dissection: a 10 -year mortality of nearly $50 \%(8,30)$.

While the conservative root repair group has better early survival on the Kaplan-Meier curve, there was no difference in the perioperative complication rates in terms of the level of distal repair. In addition, patients that underwent extensive distal surgery had a better early, mid and longterm survival, although this did not reach statistical significance. Given these observations, it seems rational to remain conservative at the level of the root, but to perform a more extensive repair at the level of the arch. Our recent development has been to perform a modified two vessel arch replacement with a "frozen elephant trunk" technique (Figure 2E,F). In experienced hands, we believe that the addition of antegrade stent grafting to a well performed hemiarch replacement is the only way to mitigate against poor long-term results. The short-term results have been superior to an isolated hemiarch approach, and this approach gives the best opportunity to improve long-term outcomes.

\section{Conclusions}

Repair of TAAD remains a challenging emergency procedure. It continues to be associated with relatively high early and late mortality rate. While numerous surgical approaches have been utilized over the years, there is accumulating evidence, including our retrospective review, that a more standardized approach should be adopted: conservative management of root pathology when feasible with valve re-suspension and limited root repair, followed by at least a hemiarch replacement. In aortic centers, the addition of antegrade stent grafting or a form of frozen elephant trunk arch replacement will likely be of long term benefit without significant added short-term risk. While we would favor central cannulation for establishment of CPB, the differences in alternative methods have not been shown to be as marked.

\section{Limitations of the study}

The current report is subject to all the limitations of a retrospective, non-randomized study biased by the 
A

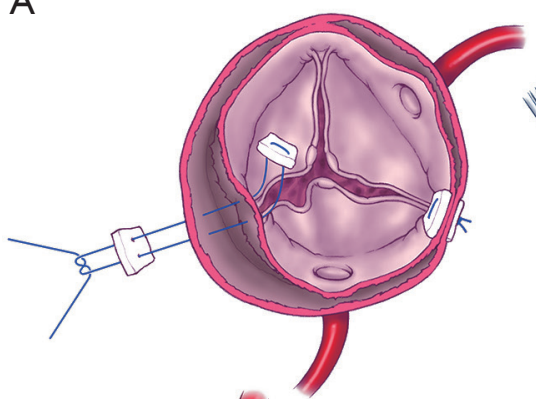

C

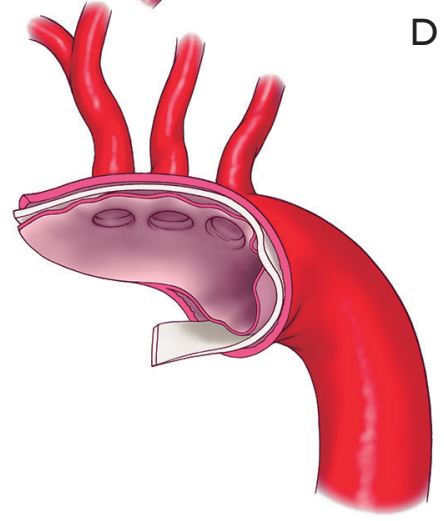

$\mathrm{E}$

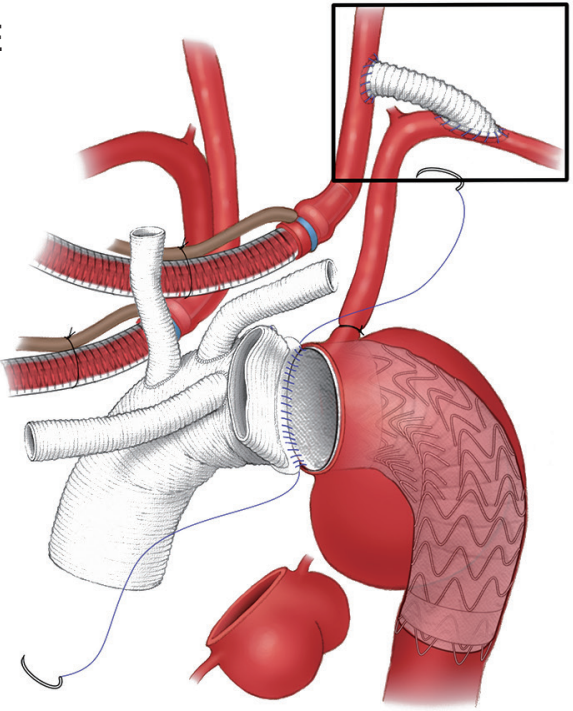

D
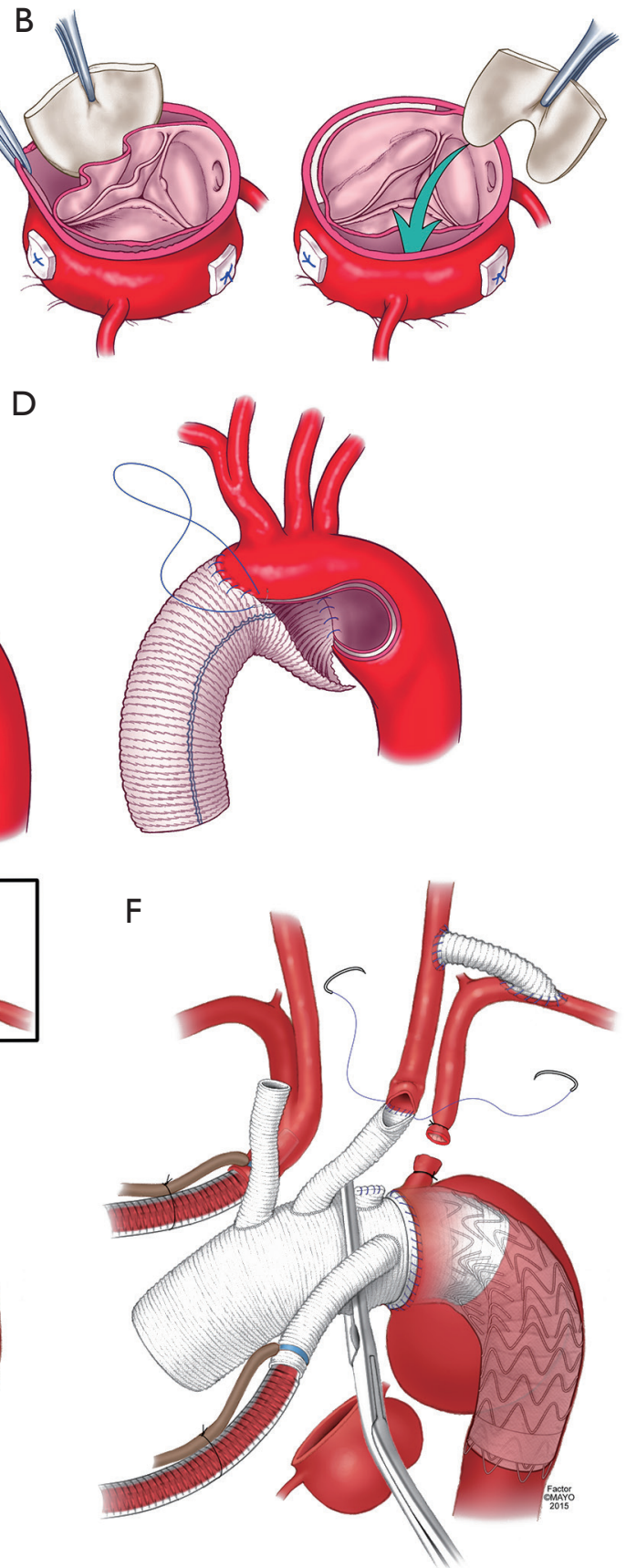

Figure 2 Proposed surgical management for type A aortic dissection. (A) Aortic valve resuspension using pledgeted mono-filament suture; (B) conservative root management by placing a felt sandwich in between the dissected layers of the root followed by tacking suture; (C,D) distal repair showing hemiarch procedure with application of felt sandwich between the dissected layers; (E,F) suggested surgical management of DeBakey type I dissection, which includes ascending aorta replacement, total arch reconstruction with antegrade deployment of frozen elephant trunk into the descending aorta. 
nature of our referral pattern, which is related to being a tertiary referral center outside a densely populated area. A randomized trial in management of this disease is not likely to ever be performed, and we have to make some conclusions on the basis of incomplete and biased studies. Data on cerebral perfusion strategy is missing on our study. Advocating for more aggressive arch replacement approach requires good cerebral protection, the details of which we were not able to evaluate in this cohort. We know that in elective total arch replacement, the systematic use of selective antegrade cerebral perfusion techniques has led to excellent neurologic outcomes.

\section{Acknowledgements}

The authors would like to express our appreciation to Katherine S. King, MS, for all the help in the data collection, statistical analysis, and manuscript editing, Ms. Rebecca Severson for helping with the data collection, and Mr. Carl Clingman for the help with the illustrations.

\section{Footnote}

Conflicts of Interest: The authors have no conflicts of interest to declare.

Ethical Statement: This study was approved by the Institutional Review Board at Mayo Clinic, Rochester MN.

\section{References}

1. Ro SK, Kim JB, Hwang SK, et al. Aortic root conservative repair of acute type A aortic dissection involving the aortic root: fate of the aortic root and aortic valve function. J Thorac Cardiovasc Surg 2013;146:1113-8.

2. Pacini D, Di Marco L, Fortuna D, et al. Acute aortic dissection: epidemiology and outcomes. Int J Cardiol 2013;167:2806-12.

3. Nishida H, Tabata M, Fukui T, et al. Surgical Strategy and Outcome for Aortic Root in Patients Undergoing Repair of Acute Type A Aortic Dissection. Ann Thorac Surg 2016;101:1464-9.

4. Bavaria JE, Brinster DR, Gorman RC, et al. Advances in the treatment of acute type A dissection: an integrated approach. Ann Thorac Surg 2002;74:S1848-52; discussion S1857-63.

5. Song MH. A learning curve in aortic dissection surgery with the use of cumulative sum analysis. Nagoya J Med Sci
2014;76:51-7.

6. Hirst AE Jr, Johns VJ Jr, Kime SW Jr. Dissecting aneurysm of the aorta: a review of 505 cases. Medicine (Baltimore) 1958;37:217-79.

7. Geirsson A, Bavaria JE, Swarr D, et al. Fate of the residual distal and proximal aorta after acute type a dissection repair using a contemporary surgical reconstruction algorithm. Ann Thorac Surg 2007;84:1955-64; discussion 1955-64.

8. Zierer A, Voeller RK, Hill KE, et al. Aortic enlargement and late reoperation after repair of acute type A aortic dissection. Ann Thorac Surg 2007;84:479-86; discussion 486-7.

9. Suzuki T, Asai T, Matsubayashi K, et al. Safety and efficacy of central cannulation through ascending aorta for type A aortic dissection. Interact Cardiovasc Thorac Surg 2010;11:34-7.

10. Niederhäuser U, Künzli A, Seifert B, et al. Conservative treatment of the aortic root in acute type A dissection. Eur J Cardiothorac Surg 1999;15:557-63.

11. Grau JB, Kuschner CE, Ferrari G, et al. Effects of a protocol-based management of type A aortic dissections. J Surg Res 2015;197:265-9.

12. Trimarchi S, Nienaber CA, Rampoldi V, et al. Contemporary results of surgery in acute type A aortic dissection: The International Registry of Acute Aortic Dissection experience. J Thorac Cardiovasc Surg 2005;129:112-22.

13. Skripochnik E, Friedman P, Michler RE, et al. The outcome of surgical management of type A aortic dissection. Asian Cardiovasc Thorac Ann 2014;22:687-93.

14. Pape LA, Awais M, Woznicki EM, et al. Presentation, Diagnosis, and Outcomes of Acute Aortic Dissection: 17Year Trends From the International Registry of Acute Aortic Dissection. J Am Coll Cardiol 2015;66:350-8.

15. Rylski B, Milewski RK, Bavaria JE, et al. Outcomes of surgery for chronic type A aortic dissection. Ann Thorac Surg 2015;99:88-93.

16. Hiratzka LF, Bakris GL, Beckman JA, et al. 2010 ACCF/ AHA/AATS/ACR/ASA/SCA/SCAI/SIR/STS/SVM guidelines for the diagnosis and management of patients with thoracic aortic disease: executive summary. A report of the American College of Cardiology Foundation/ American Heart Association Task Force on Practice Guidelines, American Association for Thoracic Surgery, American College of Radiology, American Stroke Association, Society of Cardiovascular Anesthesiologists, Society for Cardiovascular Angiography and Interventions, 
Society of Interventional Radiology, Society of Thoracic Surgeons, and Society for Vascular Medicine. Catheter Cardiovasc Interv 2010;76:E43-86.

17. Casselman FP, Tan ES, Vermeulen FE, et al. Durability of aortic valve preservation and root reconstruction in acute type A aortic dissection. Ann Thorac Surg 2000;70:1227-33.

18. Kobuch R, Hilker M, Rupprecht L, et al. Late reoperations after repaired acute type A aortic dissection. J Thorac Cardiovasc Surg 2012;144:300-7.

19. Silva J, Maroto LC, Carnero M, et al. Ascending aorta and aortic root reoperations: are outcomes worse than first time surgery? Ann Thorac Surg 2010;90:555-60.

20. Klodell CT, Karimi A, Beaver TM, et al. Outcomes for acute type A aortic dissection: effects of previous cardiac surgery. Ann Thorac Surg 2012;93:1206-12; discussion 1212-4.

21. Concistrè G, Casali G, Santaniello E, et al. Reoperation after surgical correction of acute type A aortic dissection: risk factor analysis. Ann Thorac Surg 2012;93:450-5.

22. Preventza O, Price MD, Simpson KH, et al. Hemiarch and Total Arch Surgery in Patients With Previous Repair of Acute Type I Aortic Dissection. Ann Thorac Surg 2015;100:833-8.

23. Luciani N, De Geest R, Anselmi A, et al. Results of reoperation on the aortic root and the ascending aorta.

Cite this article as: Cabasa A, Pochettino A. Surgical management and outcomes of type A dissection-the Mayo Clinic experience. Ann Cardiothorac Surg 2016;5(4):296-309. doi: 10.21037/acs.2016.06.01
Ann Thorac Surg 2011;92:898-903.

24. De Paulis R, Czerny M, Weltert L, et al. Current trends in cannulation and neuroprotection during surgery of the aortic arch in Europe. Eur J Cardiothorac Surg 2015;47:917-23.

25. Moon MR, Sundt TM 3rd, Pasque MK, et al. Does the extent of proximal or distal resection influence outcome for type A dissections? Ann Thorac Surg 2001;71:1244-9; discussion 1249-50.

26. Kallenbach K, Oelze T, Salcher R, et al. Evolving strategies for treatment of acute aortic dissection type A. Circulation 2004;110:II243-9.

27. Rice RD, Sandhu HK, Leake SS, et al. Is Total Arch Replacement Associated With Worse Outcomes During Repair of Acute Type A Aortic Dissection? Ann Thorac Surg 2015;100:2159-65; discussion 2165-6.

28. Crawford ES, Kirklin JW, Naftel DC, et al. Surgery for acute dissection of ascending aorta. Should the arch be included? J Thorac Cardiovasc Surg 1992;104:46-59.

29. Ohtsubo S, Itoh T, Takarabe K, et al. Surgical results of hemiarch replacement for acute type A dissection. Ann Thorac Surg 2002;74:S1853-6; discussion S1857-63.

30. Kim JB, Lee CH, Lee TY, et al. Descending aortic aneurysmal changes following surgery for acute DeBakey type I aortic dissection. Eur J Cardiothorac Surg 2012;42:851-6; discussion 856-7. 